Hild, Marvin (2021): „Zwischen

Schein und Sein. Wie sich nieder-

sächsische DITIB-Funktionäre in

sozialen Medien äußern" In: De-

mokratie Dialog 9 (2021) S. 43-51.

doi:10.17875/gup2021-1773

\section{Zwischen Schein und Sein}

\author{
Wie sich niedersächsische DITIB- \\ Funktionäre in sozialen Medien \\ äußern
}

Marvin Hild

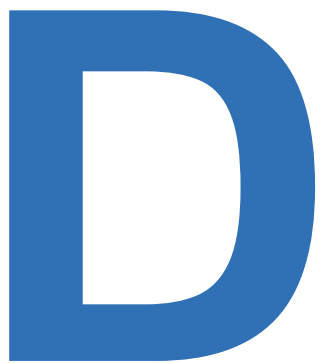

ie Türkisch Islamische Union der Anstalt für Religion e. V., kurz DITIB, bekennt in ihren Grundsätzen zur freiheitlich-demokratischen Grundordnung. Ebenso legt sie „Wert auf Freundschaftlichkeit, Achtung, Nachsicht, Toleranz und Solidarität der Menschen untereinander und gegenüber anderen Glaubensangehörigen" und lehnt jede "Art von Gewalt und Aufruf zur Gewalt"1 ab. Dieser Darstellung lief das Verhalten des ehemaligen Göttinger DITIB-Vorsitzenden, Mustafa Keskin, in den sozialen Medien jahrelang zuwider, wie die Jüdische Allgemeine am 07.07.2021 berichtete. Für einige Äußerungen muss Keskin sich sogar vor Gericht verantworten. ${ }^{2}$ Im Vorfeld der Vollversammlung des Landesju-

1 Türkisch Islamische Union der Anstalt für Religion e. V. Grundsätze, URL: https://www.ditib.de/defaultl.php?id=5\&sid=9\&lang=de [eingesehen am 27.07.2021].

2 Vgl. O. V.: Früherer Göttinger Ditib-Chef muss im Oktober vor Gericht, in: Jüdische Allgemeine, 07.07.2021, URL: https://www.juedische-allgemeine.de/religion/ gendrings Niedersachsen im März 2021, auf der über einen Antrag auf Vollmitgliedschaft des DITIB-Jugendverbands für Niedersachsen und Bremen abgestimmt werden sollte, nahm die "Sozialistische Jugend - Die Falken" in Göttingen diesen Umstand zum Anlass, den dortigen Ortsverband der DITIB genauer zu betrachten und stieß im Zuge dessen auch auf das Facebook-Profil Keskins. Dieser habe, nach dokumentierten Berichten der Falken, auf seinem Profil „antisemitische Hassbotschaften und Verschwörungsmythen verbreitet, gegen Kurden ${ }^{3}$ und Armenier [ge]hetzt und sich positiv auf islamistische Strömungen wie die Muslimbruderschaft bez[ogen]..4 Weiter-

frueherer-goettinger-ditib-chef-muss-im-oktober-vor-gericht/ [eingesehen am 07.09.2021].

3 Aus Gründen der besseren Lesbarkeit wird bei Personenbezeichnungen und personenbezogenen Hauptwörtern das generische Maskulinum verwendet. Entsprechende Begriffe gelten im Sinne der Gleichbehandlung grundsätzlich für alle Geschlechter. Die verkürzte Sprachform beinhaltet keine Wertung

4 Sozialistische Jugend - Die Falken, Ortsverband Göttingen: Vorsitzender der Göttinger Ditib-Gemeinde/ 

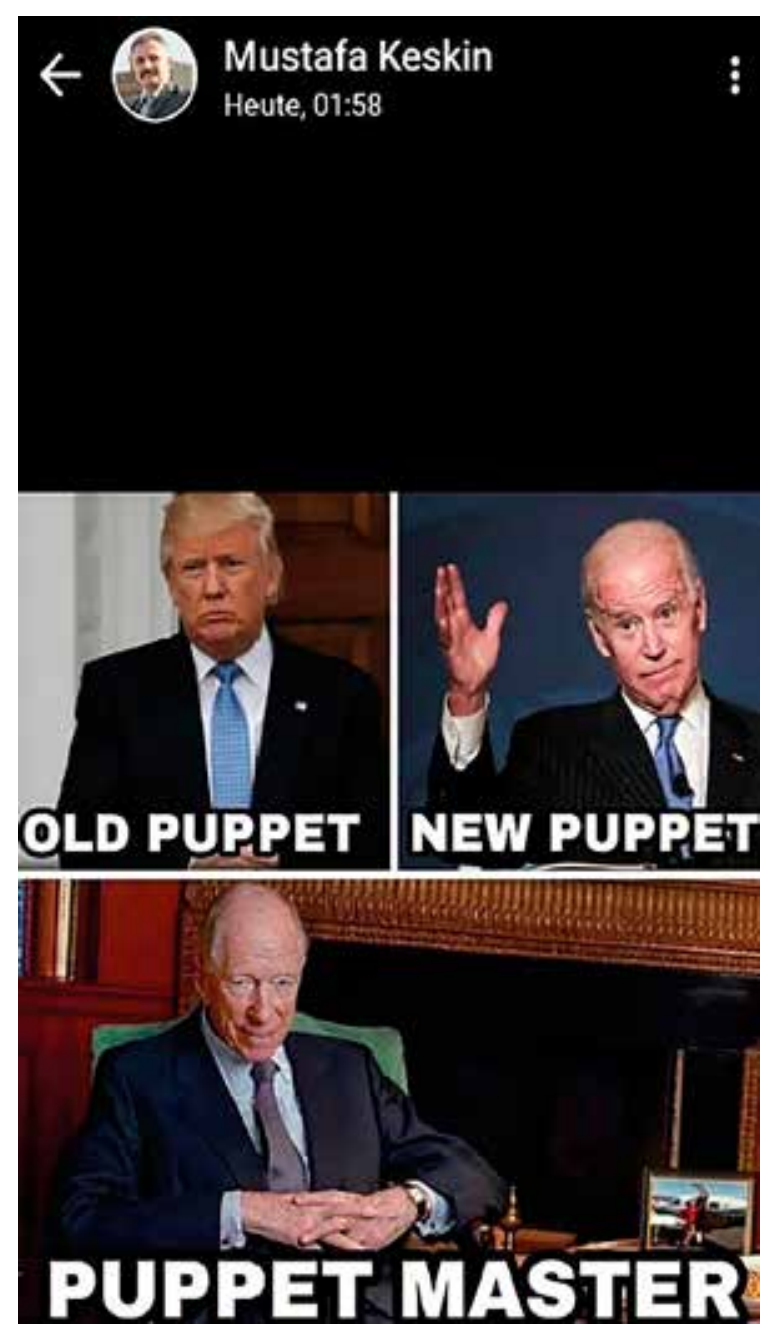

Abbildung 1: Sozialistische Jugend - Die Falken, Ortsverband Göttingen: Vorsitzender der Göttinger Ditib-Gemeinde/Türkisch Islamische Gemeinde zu Göttingen e. V. verbreitet antisemitische Verschwörungsmythen und Hassbotschaften - Stellungnahme des Göttinger Ortsverbands der Sozialistischen Jugend - Die Falken, öffentliche Mail vom 05.02.2021, S. 3.

hin konstatieren die Jungsozialisten einen „[...] türkisch-nationalistischen Grundtenor und einem positiven Bezug auf ein Groß-Osmanentum."5 Das Profil ist mittlerweile nicht mehr abrufbar und

Türkisch Islamische Gemeinde zu Göttingen e.V. verbreitet antisemitische Verschwörungsmythen und Hassbotschaften - Stellungnahme des Göttinger Ortsverbands der Sozialistischen Jugend - Die Falken, öffentliche Mail vom 05.02.2021, S. 1.

5 Ebd. die Veröffentlichung der Falken ist keine akademische Studie, sondern die Einschätzung einer politischen Gruppierung, die im Diskurs eigene Interessen verfolgt. Die Aussagen decken sich allerdings mit der beschriebenen medialen $\mathrm{Be}$ richterstattung und die mitgelieferten Zitate und Screenshots scheinen authentisch zu sein und vermitteln einen Eindruck des gefundenen Materials.

Im abgebildeten Facebook-Posting von Keskin ist in der unteren Bildhälfte Jacob Rothschild zu sehen, ${ }^{6}$ ein eindeutiger Bezug auf antisemitische Verschwörungstheorien des "Weltjudentums", welches angeblich im Verborgenen die Geschicke der Welt lenkt.' Ebenso ist eine Zeichnung zu sehen, auf der ein blutiges Kind vor einer Israelfahne liegt, während ihm der Arm eines israelischen Soldaten einen Dolch in der Form Israels in die Brust stößt. ${ }^{8}$ Israel wird hier somit als Akteur gezeichnet, der Kriegsverbrechen begeht, indem er unschuldige, wehrlose Opfer tötet. Die Falken verweisen auf weitere Funde im Zuge ihrer „Recherche im Online-Netzwerk der Moscheegemeinden"', bei denen u. a. auf türkische rechtsextreme Bewegungen, wie die Ülkücü (Graue Wölfe) und die Partei MHP, Bezug genommen werde. ${ }^{10}$ Auch bei Vorstandsmitgliedern der DITIB-Jugend habe man "Symbole der Grauen Wölfe, antiisraelische Posts und Kontakte zur Millî Görüş"" entdeckt.

Die hier vorgefundenen rechtsextremen, antisemitischen und radikalislamischen Inhalte der Postings Keskins stehen in einem deutlichen möglicherweise gar strafrechtlich relevanten -

6 Ebd., S. 3

7 Vgl. Pfahl-Traughber, Armin: Antisemitismus, in: Bozay, Kemal/Borstel, Dierk (Hrsg.): Ungleichwertigkeitsideologien in der Einwanderungsgesellschaft, Wiesbaden 2017, S. 83-102, hier S. 85 f.

8 Vgl. Die Falken: Stellungnahme, S. 4

9 Ebd., S. 2

10 Vgl. ebd., S. 2

11 Ebd., S. 2 
Spannungsverhältnis zu der proklamierten formalpolitischen Neutralität der DITIB. Damit drängt sich die Frage auf, wie sich die Funktionäre anderer DITIB-Gemeinden des Landesverbands Niedersachsen und Bremen in den sozialen Medien präsentieren und ob die Entgleisungen Keskins damit ein Einzelfall waren, die dem Integrationsprojekt nicht weiter im Wege stehen. Um dies zu untersuchen, wurden explorativ weitere niedersächsische Ortsverbände betrachtet und der forschungsleitenden Frage nachgegangen: Lassen sich bei anderen Funktionären rechtsextreme, antisemitische oder radikalislamische Inhalte mit anti-demokratischem Charakter finden?

\section{Der Facebook-Auftritt ausge- wählter DITIB-Funktionäre}

Für den Hannoverschen Ortsverband ist das Profil des Beisitzers Yunus Yazici von besonderem Interesse, ${ }^{12}$ hier fallen insbesondere Beiträge mit Israelbezug auf. ${ }^{13}$ Seine Postings sind vermutlich Reaktionen auf den Beginn der israelischen Militäraktion „Operation Protective Edge" (08.07.201426.08.2014) gewesen, mit denen Israel auf anhaltenden Beschuss durch Raketen aus dem Gazastreifen mit Artillerie- und Luftangriffen sowie einer Bodenoffensive reagierte. Die auf dem Profil am 09.07.2014 veröffentlichten Fotos zeigen tote

12 Die DITIB-Gemeinde in Hannover hat auf ihrer Website eine detaillierte Übersicht aller Mitglieder des Vorstands inklusive Fotos, was die zweifelsfreie Zuordnung der Profile ermöglichte.

13 Yazici, Yunus: Profil, in: Facebook, URL: https://www. facebook.com/yunus.yazici.92 [eingesehen am 24.05.2021]. und verstümmelte Kinderleichen, die ironisch als "terroristen [sic]" bezeichnet werden und seinem Kommentar zufolge von den "verdammten israelis [sic]" getötet wurden. ${ }^{14}$ Ebenso ist die abgebildete Karikatur vom 12.07.2014 zu sehen. ${ }^{15}$
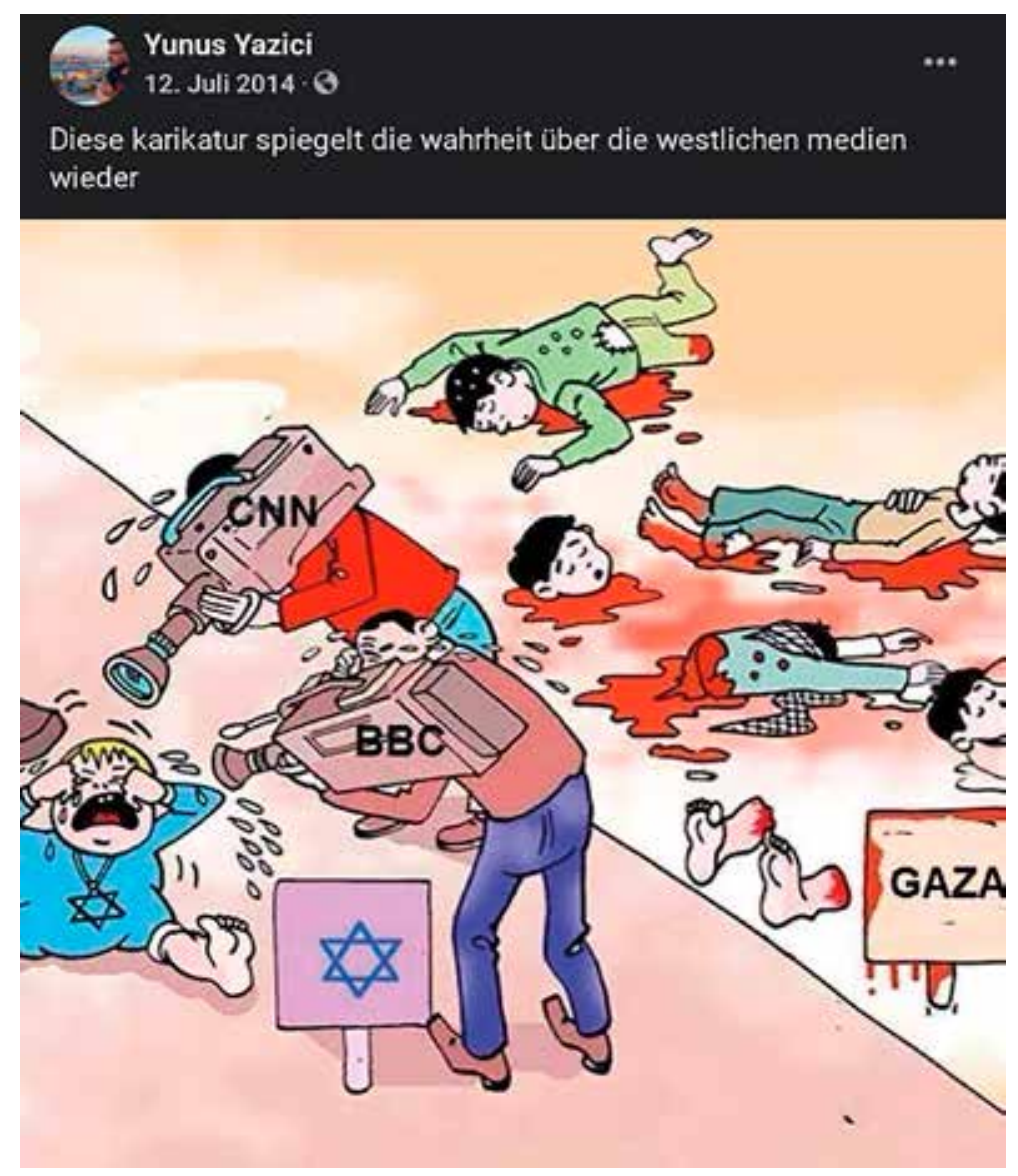

Abbildung 2: Yazici, Yunus: Facebook-Post vom 12.07.2014, in: Facebook, URL: https://www.facebook.com/photo?fbid=10202559419083321\&set=a. 2135288658398 [eingesehen am 24.05.2021].

14 Yazici, Yunus: Facebook-Post vom 09.07.2014, in: Facebook, URL: https://www.facebook.com/photo?fbid $=10202545123325936 \&$ set $=a .2135288658398$ [eingesehen am 24.05.2021]; Yazici, Yunus: Facebook-Post vom 09.07.2014, in: Facebook, URL: https://www.facebook.com/yunus.yazici.92/posts/10202545106285510 [eingesehen am 24.05.2021].

15 Yazici, Yunus: Facebook-Post vom 12.07.2014, in: Facebook, URL: https://www.facebook.com/photo?fbid=10202559419083321\&set=a.2135288658398 [eingesehen am 24.05.2021]. 
Augenfällig ist hier die verzerrende Darstellung der blutigen und zerfetzten Leichen, die unbeachtet in Gaza liegen und denen in den Rücken geschossen wurde. Das Motiv der unschuldigen, friedlichen Protestanten, denen von Soldaten der Israeli Defense Forces in den Rücken geschossen wurde, ist ein wiederkehrendes Narrativ in der einschlägigen pro-palästinensischen Berichterstattung. Der Staat Israel bzw. seine Streitkräfte werden in diesen Postings als sinistere Schurken dargestellt, die mutwillig Kriegsverbrechen gegen Zivilisten begehen, was der Delegitimierung des Staates Israel Vorschub leistet. Weiterhin erregt ein von Yazici geteiltes Video vom 25.07.2014 mit dem Titel "Stop Terrorism in Gaza" Aufmerksamkeit, das von ihm mit den Worten kommentiert ist: "Ihr verfluchten israelis [sic] | Möget ihr in der hölle [sic] schmoren | Ich verfluche euch und hitler das [sic] er euch nicht ausgerottet hat ${ }^{\prime \prime 16}$. Hier wird nicht nur eine religiöse Verfluchung ausgesprochen, sondern vor allem auch der Holocaust gutgeheißen und Bedauern darüber ausgedrückt, dass nicht alle Juden der systematischen Massenvernichtung zum Opfer gefallen sind. Im Gegensatz zu den vorangegangenen Postings, die eher israelfeindlich antisemitisch waren (sich also gegen die Existenz des jüdischen Staates richteten), geht dieser Betrag einen Schritt weiter zum eliminatorischen Antisemitismus, zur Unterstützung der systematischen Vernichtung von Juden per se.

Für den Vorstand der DITIB-Gemeinde Osnabrück sind besonders die Profile des Vorsitzenden, Ramazan Karacan, ${ }^{17}$ und eines Stellvertre-

16 Yazici, Yunus: Facebook-Post vom 25.07.2014, in: Facebook, URL: https://www.facebook.com/yunus.yazici.92 [eingesehen am 24.05.2021, zu einem späteren Zeitpunkt war das Video bereits gelöscht, das Material wurde vorher gesichert].

17 Karacan, Ramazan: Profil, in: Facebook, URL: https:// www.facebook.com/ramazan.karacan.908 [eingesehen am 24.05.2021]. ters, Muhammed Aktas, ${ }^{18}$ interessant. $^{19}$ Auf dem Profil Karacans sind mehrere Beiträge mit Bezug zur Ülkücü-Bewegung zu sehen, die in Deutschland häufig als „Graue Wölfe” bezeichnet wird. Der Name „Graue Wölfe" rekurriert auf eine türkische Sage, nach der ein grauer Wolf "in der Frühzeit den türkischen Stämmen bzw. Kriegern den Weg nach Kleinasien gewiesen haben soll“20. Ülkücü (dt.: Idealist) ist eine rechtsextreme türkische Bewegung Die türkische MHP (Partei der nationalistischen Bewegung), die der Regierungspartei AKP des Staatspräsidenten Recep Tayyip Erdoğan zur parlamentarischen Mehrheit verhilft, hat ihre ideologischen Wurzeln in der Idealisten-Bewegung. Dieser türkische Rechtsextremismus ist durch eine Synthese zwischen Islam und einem pantürkistischen Nationalismus gekennzeichnet, ${ }^{21}$ der häufig rassistisch konnotiert ist und die Abwertung nicht-türkischstämmiger Bevölkerungsgruppen (etwa von Kurden und Armeniern) rechtfertigt, ${ }^{22}$ wie sie bei Mustafa Keskin zu beobachten war Hinzu kommt eine Verherrlichung des Osmanischen Reiches. Diese Osmanen-Nostalgie findet etwa in einer roten bzw. grünen Flagge mit drei Halbmonden Ausdruck, die sich an Varianten der Osmanischen Fahne anlehnt. Die MHP verwendet

18 Aktas, Muhammed: Profil, in: Facebook, URL: https:// www.facebook.com/muhammed.aktas.12 [eingesehen am 24.05.2021]

19 Die Gemeinde Osnabrück hat zwar in ihrer Übersicht über die Vorstandsmitglieder (http://www.ditib-osnabrueck.de/der-vorstand.html [eingesehen am 23.03.2021]) lediglich Karacan mit einem Foto versehen, allerdings sind die Facebook-Profile von Karacan und Aktas befreundet, wodurch sich auch Letzteres verifizieren ließ.

20 Aslan, Fikret/Bozay, Kemal: Die Republik Türkei, in: Aslan, Fikret/Bozay, Kemal (Hrsg.): Graue Wölfe heulen wieder. Türkische Faschisten und ihre Vernetzung in Deutschland, Münster 2012, S. 43-73, hier S. 59

21 Vgl. Aslan, Fikret/Bozay, Kemal: Die türkische rechtsextreme Bewegung und ihre Ideologie nach 1990, in Aslan, Fikret/Bozay, Kemal (Hrsg.): Graue Wölfe heulen wieder. Türkische Faschisten und ihre Vernetzung in Deutschland, Münster 2012, S. 74-105, hier S. 84 f

22 Vgl. ebd., hier S. $76 \mathrm{ff}$ 
dabei die Flagge in der roten Variante, die grüne Fahne (Farbe des Islam) wird mit der „Partei der Großen Einheit" (BBP) assoziiert, die sich in den 1990er Jahren von der MHP abspaltete und einen islamistischen Einschlag hat. $^{23}$ Ebendiese Symbolik ist in Ramazan Karacans Facebook-Profil zu sehen. So teilte er am 16.05.2016 ein Foto mit einem Logo der Grauen Wölfe. ${ }^{24}$ Auf einem Profilbild seines Bruders Bilal aus dem Jahr 2015, auf dem er verlinkt wurde, steht Karacan vor einer roten Wand, auf der drei große weiße Halbmonde prangen. $^{25}$ Zwischen Januar und März 2014 teilte er einen Beitrag der rechtsextremen MHP sowie drei Beiträge des ihr nahestehenden Accounts „Fatih Sultan Mehmed (The Conqueror)"; auf einem davon vom 09.01.2014 sind $u$. a. eine grüne und eine rote Fahne mit jeweils drei Halbmonden zu erkennen. ${ }^{26}$ Diese Postings sind beispielhaft dafür, was Fikret Aslan und Kemal Bozay mit der "Türkisch-islamische[n] Synthese"27 bzw. Tanıl Bora mit "Turko-Islam"28 im Kontext der Ülkücü-Bewegung meinen. Emre Arslan weist darauf hin, dass die drei Halbmonde auf grünem Hintergrund von

23 Vgl. Arslan, Emre: Graue Wölfe: Zwischen Herrschernation, Mythos und Symbolik, in: Aslan, Fikret/Bozay, Kemal (Hrsg.): Graue Wölfe heulen wieder. Türkische Faschisten und ihre Vernetzung in Deutschland, Münster 2012, S. 123-132, hier S. 127

24 Karacan, Ramazan: Facebook-Post vom 16.05.2016, in: Facebook, URL: https://www.facebook.com/ramazan. karacan.908/posts/1163357573684021 [eingesehen am 24.05.2021]

25 Karacan, Ramazan: Facebook-Post vom 25.05.2016, in: Facebook, URL: https://www.facebook.com/ramazan. karacan.908/posts/1168861049800340 [eingesehen am 24.05.2021].

26 Karacan, Ramazan: Facebook-Post vom 09.01.2014, in: Facebook, URL: https://www.facebook.com/ramazan. karacan.908/posts/706932599326523 [eingesehen am 24.05.2021]

27 Aslan/Bozay: Die türkische rechtsextreme Bewegung und ihre Ideologie nach 1990, S. 78.

28 Bora, Tanil: Nationalist Discourses in Turkey, in: The South Atlantic Quarterly, Jg. 102 (2003), H. 2/3, S. 433451, hier S. 449 islamistischen Ülkücüs als Symbol genutzt werden. $^{29}$

Für die Forschungsfrage dieses Artikels relevant ist zudem ein Video der Seite "Kastamonuluyum" (dt.: Ich komme aus Kastamonu) vom 20.11.2014, das Ramazan Karacan geteilt hat und das unter anderem antisemitische bzw. Israel dämonisierende Darstellungen enthält. So ist dort der Davidstern aus Stacheldraht auf blutbefleckter israelischer Fahne zu sehen, in der Mitte ein Gesicht mit einem Vampirgebiss. ${ }^{30}$ Die Darstellung von Juden als Vampire ist als antisemitisches Motiv nicht unüblich und kann mit der Vorstellung von Juden als raffgierigen Kapitalisten ${ }^{\mathbf{3 1}}$ oder der Legende, Juden würden nicht-jüdische Kinder ermorden, um Mazzen mit deren Blut zu backen, ${ }^{32}$ in Zusammenhang stehen.

Muhammed Aktas hingegen teilte auf seinem Facebook-Profil Beiträge verschwörungstheoretischer Nachrichtenportale, die Bezug auf den Putschversuch in der Türkei 2016 nehmen. Am 18.07.2016 teilte er einen Artikel des Contra Magazins mit dem Titel "WikiLeaks: Wurden die Putschisten in der Türkei von der CIA unterstützt?" und am 09.01.2017 einen weiteren von Nachrichtenexpress: „Bericht: NATO versprach PKK Sturz Erdogans".33 Der zweite Artikel erwähnt Verbindungen des Islamischen Staats, der kurdischen

29 Vgl. Arslan: Graue Wölfe, S. 127 f.

30 Karacan, Ramazan: Facebook-Post vom 20.11.2014, in: Facebook, URL: https://www.facebook.com/ramazan. karacan.908/posts/869447906408324 [eingesehen am 24.05.2021].

31 Vgl. Pfahl-Traughber: Antisemitismus, S. 85.

32 Vgl. Baum, Steven/Rudski, Jeffrey: Research Note: Anti-Semitism and Superstition, in: Journal of Contemporary Religion, Jg. 23 (2008), H. 1, S. 77-86, hier S. 77.

33 Aktas, Muhammed: Facebook-Post vom 18.07.2016, in: Facebook, URL: https://www.facebook.com/muhammed.aktas.12/posts/1150518828344913 [eingesehen am 24.05.2021]; Aktas, Muhammed: Facebook-Post vom 09.01.2017, in: Facebook, URL: https://www.facebook. com/muhammed.aktas.12/posts/1317431898320271 [eingesehen am 24.05.2021]. 


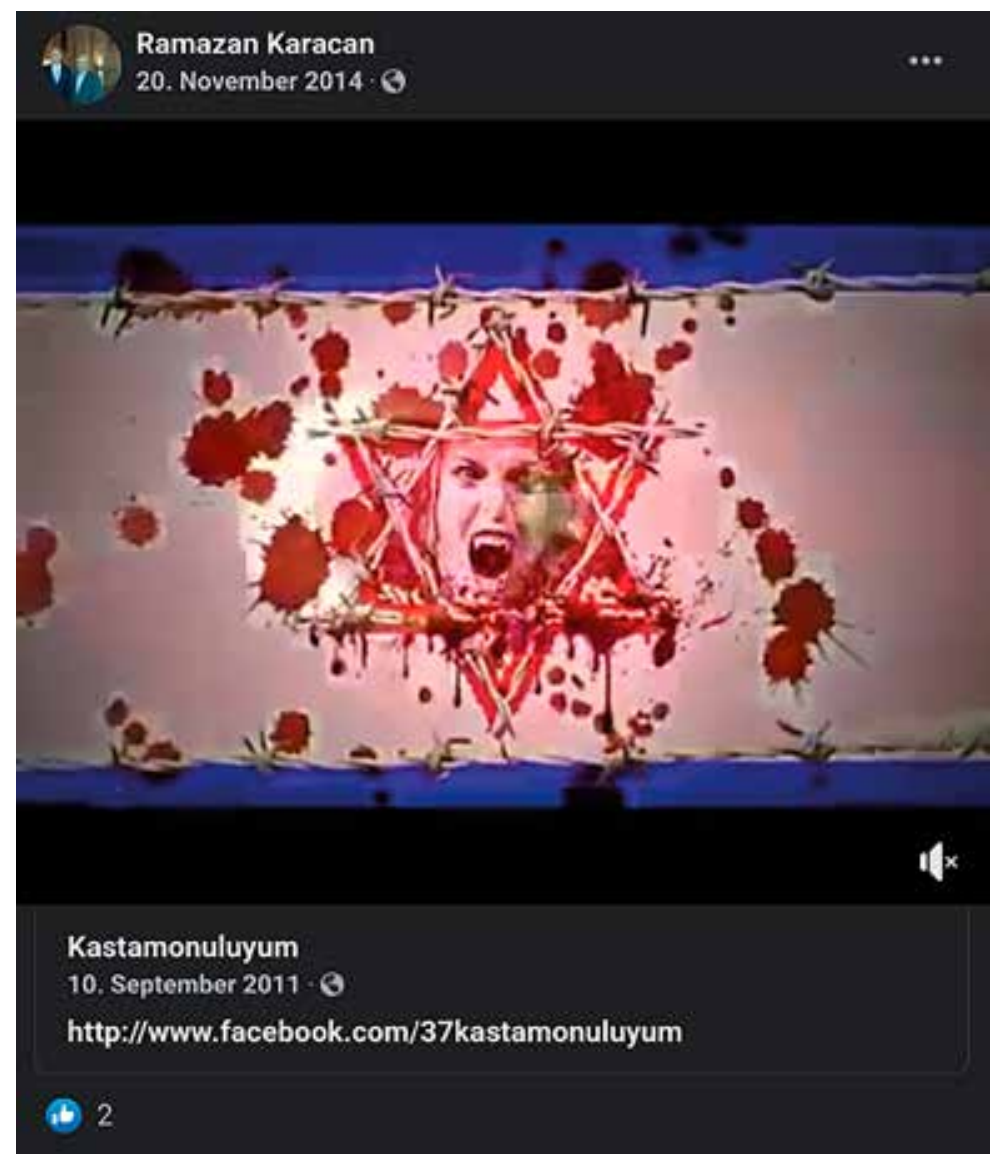

Abbildung 3: Karacan, Ramazan: FacebookPost vom 20.11.2014, in: Facebook, URL https://www.facebook.com/ramazan karacan.908/posts/869447906408324 [eingesehen am 24.05.2021].

wisse Schnittmengen gibt, etwa (israelbezogenen) Antisemitismus oder, wie in diesem Fall, Anti-Amerikanismus. ${ }^{36}$

Für die Betrachtung der DITIB-Gemeinde Hildesheim ${ }^{37}$ ist das Facebook-Profil des örtlichen Buchhalters und Kassenwarts Hakan Karaduman von besonderer Bedeutung. ${ }^{38}$ Hier findet sich in einem Beitrag vom 22.03.2021 die oben beschriebene Symbolik des islamistischen Teils der Ülkücü-Bewegung. Außerdem wird in weiteren Posts der Säkularismus abgelehnt und Sympathie für eine auf der Scharia fußende Gesellschaftsordnung ausgedrückt. Ein

Arbeiterpartei PKK und der Gülen-Bewegung, die miteinander hinsichtlich des Putschversuchs konspiriert haben sollen. ${ }^{34}$ Hier wird also die Verschwörungstheorie kolportiert, dass verschiedene Akteure mit gänzlich unterschiedlichen Ideologien gemeinsam daran arbeiten würden, der Türkei zu schaden. In Bezug auf das Contra Magazin ist anzumerken, dass es sich hier eigentlich um ein Medium aus dem Spektrum des österreichischen Rechtspopulismus handelt, passende Artikel, die das eigene Weltbild bestätigen jedoch durchaus auch bei manchen Muslimen Anklang finden. ${ }^{35}$ Dies lässt sich dadurch erklären, dass es zwischen islamistischer und rechtsextremer Ideologie ge-

34 Vgl. NEX24: Bericht: NATO versprach PKK Sturz Erdogans, in: NEX24 News, 08.01.2017, URL: https://nex24. news/2017/01/bericht-nato-versprach-pkk-sturz-erdogans/ [eingesehen am 27.07.2021].

35 Vgl. Klevesath, Lino et al:: Radikalislamische YouTube-Propaganda. Eine qualitative Rezeptionsstudie unter jungen Erwachsenen, Bielefeld 2021, S. 69
Post vom 18.04.2021 in türkischer Sprache sagt beispielsweise Folgendes aus: „Wenn ich gegen Säkularismus bin und deswegen als Atatürks Feind bezeichnet werde, seid ihr Allahs Feinde, wenn ihr gegen die Scharia seid." ${ }^{\prime 39}$ Ein weiterer

36 Vgl. Meiering, David et al.: Brückennarrative - Verbindende Elemente für die Radikalisierung von Gruppen, PRIF Report 7, Frankfurt am Main 2018, S. 13 f.

37 Auch die DITIB-Gemeinde in Hildesheim hat eine bebilderte Übersicht der Vorstandsmitglieder (https:// selimiyecamii-hildesheim.com/yonetim-kurulu/ [eingesehen am 23.03.2021]), sodass sich die Zuordnung der Social-Media-Profile verifizieren ließ.

38 Karaduman, Hakan: Profil, in: Facebook, URL: https:// www.facebook.com/hakan.karaduman.3954 [eingesehen am 27.06.2021].

39 Karaduman, Hakan: Facebook-Post vom 18.04.2021, in: Facebook, URL: https://www.facebook.com/hakan.karaduman.3954/posts/4240553725974899 [eingesehen am 27.06.2021; eigene Übersetzung des türkischen Originals]. 
Post vom 23.04.2021 lautet: „Der Staat wird nicht vom Säkularismus regiert, sondern von den Versen Allahs, die wir vom Rasulullah [Mohammed, Anm. d. Verf.] kennen." ${ }^{40}$ Am 10.04.2021 teilte Karaduman ein Bild mit dem Titel „Die Verfassung, die Muslime wollen"41. Diese Verfassung bestehe aus folgenden vier Punkten: „1. Die Souveränität gehört bedingungslos Allah. | 2. Die Regierungsform ist der Islam. | 3. Sie nimmt ihre Verbote aus dem Koran und der Sunna. | 4. Eine Änderung der ersten 3 Artikel ist nicht möglich." Das für die Demokratie zentrale Prinzip der Volkssouveränität wird hier somit explizit abgelehnt.

Schließlich finden sich auch israelbezogen und eliminatorisch antisemitische Inhalte bei Karaduman. So gibt es auf seinem Profil ein Bild und ein geteiltes Video vom 10.05.2021 respektive 09.05.2021, in denen Israel als "Terrorist"42 bzw. „terroristischer Staat"43 bezeichnet wird - hierbei wird ein schon mehrfach geäußertes Zitat des türkischen Präsidenten aufgegriffen. ${ }^{44}$ Weiterhin findet sich ein Beitrag, der den Tag herbeifantasiert, an dem die Türkei die Zionisten für ihre Landnahme an den Palästinensern bestrafen

40 Karaduman, Hakan: Facebook-Post vom 23.04.2021, in: Facebook, URL: https://www.facebook.com/hakan.karaduman.3954/posts/4254406904589581 [eingesehen am 27.06.2021; eigene Übersetzung des türkischen Originals].

41 Karaduman, Hakan, Facebook-Post vom 10.04.2021, in: Facebook, URL: https://www.facebook.com/photo?fbid=4216804165016522\&set=a.655755757788065 [eingesehen am 27.06.2021; eigene Übersetzung des türkischen Originals].

42 Karaduman, Hakan, Facebook-Post vom 10.05.2021, in: Facebook, URL: https://www.facebook.com/photo?fbid $=4305112662852338 \&$ set $=a .655755757788065$ [eingesehen am 27.06.2021]

43 Karaduman, Hakan, Facebook-Post vom 09.05.2021, in: Facebook, URL: https://www.facebook.com/hakan.karaduman.3954/posts/4300444373319167 [eingesehen am 27.06.2021]

44 Vgl. Bellut, Daniel Derya/Köylü, Hilal: Nahostkonflikt: Erdogan nennt Israel „Terrorstaat", in: DW, 18.05.2021, URL: https://p.dw.com/p/3tYkf [eingesehen am 27.07.2021]. werde $^{45}$ sowie ein angebliches Zitat von Jacob Rothschild, gepostet am 15.04.2021, in dem die Türken als "das scharfe Schwert des Islam" bezeichnet werden, das Israel auslöschen und nach Wien vorrücken werde. ${ }^{46}$ Rothschild als Angehöriger einer weltweit vertretenen jüdischen Bankiersfamilie steht hier stellvertretend für das Weltjudentum und Israel als imperialistisches Projekt. Mindestens in dem letztgenannten Beitrag ist mit der Vernichtungsfantasie die Schwelle vom den Staat Israel delegitimierenden zum eliminatorischen Antisemitismus überschritten.

\section{Résumé}

Die stichprobenartige vergleichende Betrachtung verschiedener DITIB-Funktionäre zeigt, dass Mustafa Keskin kein Einzelfall und seine türkisch-rechtsextremen und antisemitischen Ansichten kein singuläres Phänomen im Landesverband Niedersachsen und Bremen sind. Da in mehreren Gemeinden die Vorstände nicht zu ermitteln waren und andernorts viele Mitglieder nur private Facebook-Profile betreiben, kann diese Darstellung selbstverständlich keinen Anspruch auf Repräsentativität erheben oder als Beleg für strukturelle Missstände gelten. Einige der gesichteten Profile enthielten keine rechtsextremen, antisemitischen oder radikalislamischen Postings mit anti-demokratischem Charakter, in mehreren Gemeinden gab es jedoch Fälle, die ähnlich oder eventuell sogar extremer gelagert waren als der Fall Keskin. Auch wenn die DITIB offiziell politisch unabhängig ist, ist sie institutionell von der türkischen Religionsbehörde und damit dem tür-

45 Karaduman, Hakan: Facebook-Post vom 09.05.2021, in: Facebook, URL: https://www.facebook.com/photo/?fbid $=4300398093323795 \&$ set $=$ a. 655755757788065 [eingesehen am 27.06.2021]

46 Karaduman, Hakan: Facebook-Post vom 15.04.2021, in: Facebook, URL: https://www.facebook.com/photo?fbid $=4230526743644264 \&$ set $=$ a. 655755757788065 [eingesehen am 27.06.2021]. 
kischen Staat abhängig; ${ }^{47}$ viele Funktionäre des Verbandes tragen die autoritäre Entwicklung der Türkei inklusive der zunehmenden Einschränkung der Pressefreiheit und anderer Grundrechte mit. Dies legt nahe, dass es zumindest weitere Fälle entsprechender Äußerungen geben könnte, was dem offiziellen Selbstverständnis des Dachverbandes als Fürsprecher von Achtung, Toleranz und Solidarität sowie dem Bekenntnis zur freiheitlich-demokratischen Grundordnung und der Ablehnung von Gewalt deutlich entgegensteht. ${ }^{48}$ Die DITIB verweist im Kontext solcher Postings lediglich auf die satzungsgemäßen „interne[n] Maßnahmen und Möglichkeiten, entsprechend mit Abmahnungen bis hin zum Ausschluss von Mitgliedern zu reagieren." ${ }^{49}$

47 Vgl. Lemmen, Thomas: Islamische Vereine und Verbände in Deutschland, Friedrich-Ebert-Stiftung, Bonn 2002, S. $34 \mathrm{f}$

48 Vgl. Türkisch Islamische Union der Anstalt für Religion e. V.: Grundsätze.

49 O. V.: DITIB: „Wir sind politisch neutral”, in: NDR Panorama, 23.03.2017, URL: https://daserste.ndr.de/panorama/aktuell/DITIB-Wir-sind-politisch-neutral,ditib156. html [eingesehen am 07.09.2021].

\section{Literatur:}

Arslan, Emre: Graue Wölfe: Zwischen Herrschernation, Mythos und Symbolik, in: Aslan, Fikret/Bozay, Kemal (Hrsg.): Graue Wölfe heulen wieder. Türkische Faschisten und ihre Vernetzung in Deutschland, Münster 2012, S. 123-132.

Aslan, Fikret/Bozay, Kemal: Die Republik Türkei, in: Aslan, Fikret/Bozay, Kemal (Hrsg.): Graue Wölfe heulen wieder. Türkische Faschisten und ihre Vernetzung in Deutschland, Münster 2012, S. 43-73.

Aslan, Fikret/Bozay, Kemal: Die türkische rechtsextreme Bewegung und ihre Ideologie nach 1990, in: Aslan, Fikret/ Bozay, Kemal (Hrsg.): Graue Wölfe heulen wieder. Türkische Faschisten und ihre Vernetzung in Deutschland, Münster 2012 S. 74-105.

Baum, Steven/Rudski, Jeffrey: Research Note: Anti-Semitism and Superstition, in: Journal of Contemporary Religion, Jg. 23 (2008), H. 1, S. 77-86.

Bellut, Daniel Derya/Köylü, Hilal: Nahostkonflikt: Erdogan nennt Israel "Terrorstaat", in: DW, 18.05.2021, https://p.dw. com/p/3tYkf [zuletzt abgerufen am 27.07.2021].

Bora, Tanıl: Nationalist Discourses in Turkey, in: The South Atlantic Quarterly, Jg. 102 (2003), H. 2/3, S. 433-451.

Klevesath, Lino/Munderloh, Annemieke/Sprengeler, Joris/ Grahmann, Florian/Reiter, Julia: Radikalislamische YouTube-Propaganda. Eine qualitative Rezeptionsstudie unter jungen Erwachsenen, Bielefeld 2021.

Lemmen, Thomas: Islamische Vereine und Verbände in Deutschland, Friedrich-Ebert-Stiftung, Bonn 2002

Meiering, David/Dziri, Aziz/Foroutan, Naika/Teune, Simon/ Lehnert, Esther/Abu Taam, Marwano: Brückennarrative - Verbindende Elemente für die Radikalisierung von Gruppen, PRIF Report 7, Frankfurt am Main 2018

O. V.: DITIB: „Wir sind politisch neutral“, in: NDR Panorama, 23.03.2017, URL: https://daserste.ndr.de/panorama/aktuell/ DITIB-Wir-sind-politisch-neutral,ditibl56.html [eingesehen am 07.09.2021].

Pfahl-Traughber, Armin: Antisemitismus, in: Bozay, Kemal/ Borstel, Dierk (Hrsg.): Ungleichwertigkeitsideologien in der Einwanderungsgesellschaft, Wiesbaden 2017, S. 83-102.

\section{Quellen:}

Aktas, Muhammed: Facebook-Post vom 18.07.2016, in: Facebook, URL: https://www.facebook.com/muhammed.aktas.12/ posts/1150518828344913 [eingesehen am 24.05.2021].

Aktas, Muhammed: Facebook-Post vom 09.01.2017, in: Facebook, URL: https://www.facebook.com/muhammed.aktas.12/ posts/1317431898320271 [eingesehen am 24.05.2021].

Aktas, Muhammed: Profil, in: Facebook, URL: https://www. facebook.com/muhammed.aktas.12 [eingesehen am 24.05.2021].

Karacan, Ramazan: Facebook-Post vom 09.01.2014, in: Facebook, URL: https://www.facebook.com/ramazan.karacan.908/ posts/706932599326523 [eingesehen am 24.05.2021].

Karacan, Ramazan: Facebook-Post vom 20.11.2014, in: Facebook, URL: https://www.facebook.com/ramazan.karacan.908/ posts/869447906408324 [eingesehen am 24.05.2021].

Karacan, Ramazan: Facebook-Post vom 16.05.2016, in: Facebook, URL: https://www.facebook.com/ramazan.karacan.908/ posts/1163357573684021 [eingesehen am 24.05.2021].

Karacan, Ramazan: Facebook-Post vom 25.05.2016, in: Facebook, URL: https://www.facebook.com/ramazan.karacan.908/ posts/1168861049800340 [eingesehen am 24.05.2021]. 
Karacan, Ramazan: Profil, in: Facebook, URL: https://www. facebook.com/ramazan.karacan.908 [eingesehen am 24.05.2021]

Karaduman, Hakan: Facebook-Post vom 10.04.2021, in: Facebook, URL: https://www.facebook.com/photo?fbid $=4216804165016522 \&$ set $=a .655755757788065$ [eingesehen am 27.06.2021]

Karaduman, Hakan: Facebook-Post vom 15.04.2021, in: Facebook, URL: https://www.facebook.com/photo?fbid $=4230526743644264 \&$ set $=a .655755757788065$ [eingesehen am 27.06.2021].

Karaduman, Hakan: Facebook-Post vom 18.04.2021, in: Facebook, URL: https://www.facebook.com/hakan.karaduman.3954/posts/4240553725974899 [eingesehen am 27.06.2021]

Karaduman, Hakan: Facebook-Post vom 23.04.2021, in: Facebook, URL: https://www.facebook.com/hakan.karaduman.3954/posts/4254406904589581 [eingesehen am 27.06.2021]

Karaduman, Hakan: Facebook-Post vom 09.05.2021, in Facebook, URL: https://www.facebook.com/hakan.karaduman.3954/posts/4300444373319167 [eingesehen am 27.06.2021]

Karaduman, Hakan: Facebook-Post vom 10.05.2021, in: Facebook, URL: https://www.facebook.com/photo?fbid $=4305112662852338 \&$ set $=a .655755757788065$ [eingesehen am 27.06.2021]

Karaduman, Hakan: Profil, in: Facebook, URL: https://www. facebook.com/hakan.karaduman.3954 [eingesehen am 27.06.2021]

NEX24: Bericht: NATO versprach PKK Sturz Erdogans, in: NEX24 News, 08.01.2017, URL: https://nex24.news/2017/01/ bericht-nato-versprach-pkk-sturz-erdogans/ [eingesehen am 27.07.2021]

O. V.: Früherer Göttinger Ditib-Chef muss im Oktober vor Gericht, in: Jüdische Allgemeine, 07.07.2021, URL: https:// www.juedische-allgemeine.de/religion/frueherer-goettinger-ditib-chef-muss-im-oktober-vor-gericht/ [eingesehen am 07.09.2021]

Sozialistische Jugend - Die Falken, Ortsverband Göttingen: Vorsitzender der Göttinger Ditib-Gemeinde/Türkisch Islamische Gemeinde zu Göttingen e.V. verbreitet antisemitische Verschwörungsmythen und Hassbotschaften - Stellungnahme des Göttinger Ortsverbands der Sozialistischen Jugend Die Falken, öffentliche Mail vom 05.02.2021

Türkisch Islamische Union der Anstalt für Religion e.V.: Grundsätze, URL: https://www.ditib.de/default1.php?id=5\&sid=9\&lang=de [eingesehen am 27.07.2021].

Yazici, Yunus: Facebook-Post vom 09.07.2014, in: Facebook, URL: https://www.facebook.com/photo?fbid $=10202545123325936 \&$ set $=a .2135288658398$ [eingesehen am 24.05.2021].

Yazici, Yunus: Facebook-Post vom 09.07.2014, in: Facebook, URL: https://www.facebook.com/yunus.yazici.92/ posts/10202545106285510 [eingesehen am 24.05.2021]

Yazici, Yunus: Facebook-Post vom 12.07.2014, in: Facebook, URL: https://www.facebook.com/photo?fbid $=10202559419083321 \&$ set $=a .2135288658398$ [eingesehen am 24.05.2021].

Yazici, Yunus: Facebook-Post vom 25.07.2014, in: Facebook, URL: https://www.facebook.com/yunus.yazici.92 [eingesehen am 24.05.2021].

Yazici, Yunus: Profil, in: Facebook, URL: https://www.facebook com/yunus.yazici.92 [eingesehen am 24.05.2021].

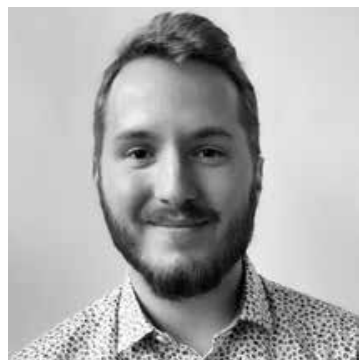

Marvin Hild (B. A.) studiert an der Georg-August-Universität Göttingen Globale Politik und Arabistik/Islamwissenschaft im Master. Seit Januar 2021 arbeitet er im Bereich Radikaler Islam des Projekts FoDex am Institut für Demokratieforschung. Seine Schwerpunkte liegen in den Bereichen radikalislamische/islamistische Ideologie, Radikalisierung und Vernetzung sowie Terrorismus(-abwehr). 